\title{
A Case Report of Morphea in a 2 Years Old Child
}

\section{Edwin Dias $^{1 *}$, Deepak K ${ }^{2}$, Vijeta Rai ${ }^{3}$ and Sahithi Chapalamadugu ${ }^{4}$}

${ }^{1}$ Professor and HOD, Department of Pediatrics, Srinivas Institute of Medical Science and Research Centre, Mangalore, India

${ }^{2}$ Junior Resident, Department of Pediatrics, Srinivas Institute of Medical Science and Research Centre, Mangalore, India

${ }^{3}$ Associate Professor, Department of Dermatology, Srinivas Institute of Medical

Science and Research Centre, Mangalore, India

${ }^{4}$ Assistant Professor, Department of Dermatology, Srinivas Institute of Medical

Science and Research Centre, Mangalore, India

*Corresponding Author: Edwin Dias, Professor and HOD, Department of Pediatrics,

Srinivas Institute of Medical Science and Research Centre, Mangalore, India.
Received: March 11, 2021

Published: March 25, 2021

(C) All rights are reserved by Edwin Dias., et al.

\begin{abstract}
Introduction: Morphea or Juvenile localized scleroderma is an autoimmune, inflammatory, chronic, slowly progressive connective tissue disease of unknown cause that preferably affects skin and underlying tissues, ultimately leading to scar like sclerosis. The incidence of Morphea ranges from 0.4 - 2.7 cases per 1,00,000 population. Treatment depends on disease subtypes, depth of involvement, stage and potential for complication.

Objective: To report a case of juvenile localised scleroderma in a 2 years old male baby.

Clinical Case: The case to be presented is of a 2 year old male child who had presented with multiple thick hypo pigmented patches of varying size over abdomen and scalp. Child had developed itchy white patch over 3 months duration, with the largest one measuring $8 \mathrm{~cm} \times 10 \mathrm{~cm}$.

Topical steroid was initially prescribed, which was stopped after a week as there was no improvement and itching was persisting. Symptom of pruritis increased gradually over the lesions and resulted in excoriations, self-mutilation and bleeding. Similar complaints were present 6 months prior, which subsided on its own. Immunized as per age. Developmental milestones were appropriate for age. No similar history in the family members.

Biopsy was done and diagnosed with Morphea. Initially patient was treated with topical steroids and topical tacrolimus, for a period of three weeks, which showed no improvement.

Conclusion: Although Morphea causes functional and aesthetic impairment, but it is rarely life-threatening. Morphea may be selflimited, but frequently has a remitting relapsing or chronic course producing significant disease burden over time. The final response to the treatment was positive in this patient.
\end{abstract}

Keywords: Morphea; Localized Scleroderma; Hypo Pigmented Patch; Alopecia; ANA Profile

\section{Introduction}

Morphea, which is also known as localized scleroderma, is a skin disorder characterized by an excessive deposition of colla- gen, leading to thickening of the, subcutaneous tissues, dermis or both, forming plaques or nodules. They are typically found on the anterior or posterior upper trunk and the arms $[1,2]$. It can be as- 
sociated with fibrosis of the internal organs, more commonly it is seen in the esophagus and lungs [1,2]. An abnormal metabolism of collagen and autoimmune phenomenon are considered to be the fundamental features of scleroderma. There is overproduction of collagen by fibroblasts is common to all forms of Morphea, particularly types I and III, in affected tissues. The mechanism by which these fibroblasts are activated is still unknown. As per the current consensus, endothelial cell injury is attributed to be the inciting event in the pathogenesis of Morphea.

Morphea is generally classified into linear, circumscribed, generalized, and pansclerotic subtypes with respect to the clinical presentation and depth of the tissue involvement [3]. Morphea can also present with extracutaneous manifestations, like fever, arthralgias, fatigue, lymphadenopathy, involvement of central nervous system. Blood investigations may reveal eosinophilia, polyclonal hyper gammaglobulinemia, and positive antinuclear antibodies. An autoimmune component is reinforced by the frequent presence of autoantibodies in individuals with Morphea, and also the association of Morphea with other autoimmune diseases like type 1 diabetes, vitiligo, systemic lupus erythematosus, and autoimmune thyroiditis [4]. Though it's rarely life threatening, an early diagnosis of Morphea and treatment is crucial to minimize potential sequel.

We report a case of Morphea in a 2-year-old boy and review the clinical and histopathological presentation, and treatment of this disease.

\section{Clinical Case}

A 2 year old boy with a history of total hair loss over a small patch in the scalp and hypo pigmented patches on the trunk and scalp for a period of 3 months presented for the evaluation to a general practitioner. The patches were situated in the left lateral side of the trunk and around the umbilicus. The patient also had itching over the patches. On initial presentation, the patient was treated with Topical Clobetasone propionate. The application was discontinued after a week as there was no changes in the lesions and persistence of the itching. Following this, Calamine lotion was prescribed, upon which the lesions turned hyperpigmented at certain regions in the lesion. Gradually pruritus increased over the lesions and resulted in multiple excoriations and bleeding. There were similar complaints 6 months previous to the present complaints, which were less severe and self-limited. Developmental milestones were appropriate for age. History of pica was present. The child had no other health complaints.

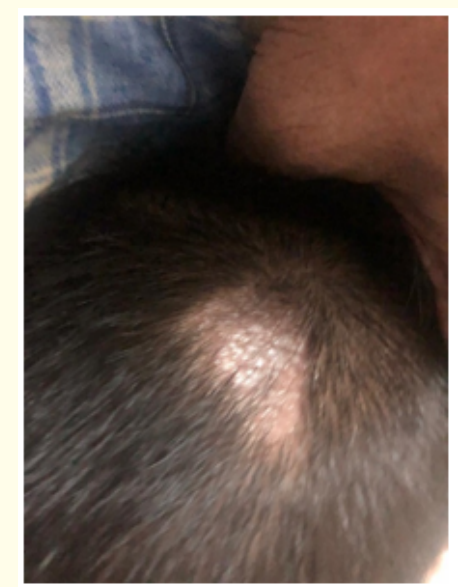

Figure 1: Patchy hair loss with hypo pigmented lesion on the scalp.

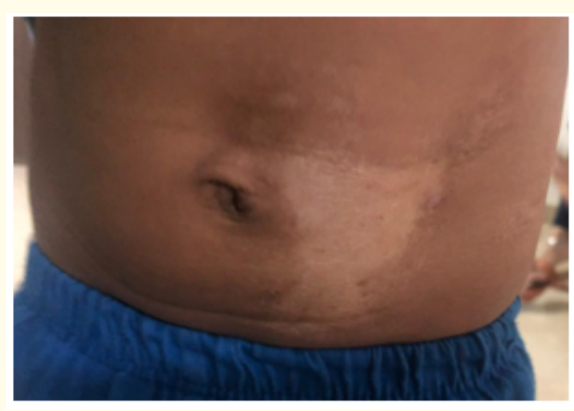

Figure 2: Hypo pigmented patch around umbilicus.

On examination: The patient had multiple hypo pigmented plaques over the scalp and abdomen, surrounded by hyperpigmentation. The largest lesion on the trunk measured around $8 \mathrm{x}$ $10 \mathrm{cms}$ at the largest dimensions. Lesions were hard in consistency with well-defined borders, irregular margins. Surrounding skin was normal. Lesion on the Scalp associated with excoriation marks and bleeding spots. The alopecia was measuring around $5 \times 2.5 \mathrm{~cm}$, with a hypo pigmented patch on the underlying area. But the rest of the scalp had normal skin and hair. The child didn't have any signs of Raynaud's syndrome or Benign Hypermobility Joint Syndrome, 
which usually accompany Morphea Differential diagnosis arrived were Morphea, Mixed connective tissue disorders, Incontinentia Pigmenti and Scleroderma.

Initial routine blood investigations like complete blood count, ESR, CRP, Serum uric acid results and urine routine results were insignificant. But ANA profile was strongly positive (+++) and Punch Biopsy of lesion revealed atrophic epidermis, collagen deposition of dermis, plasma cell and lymphocyte infiltration at perivascular regions.

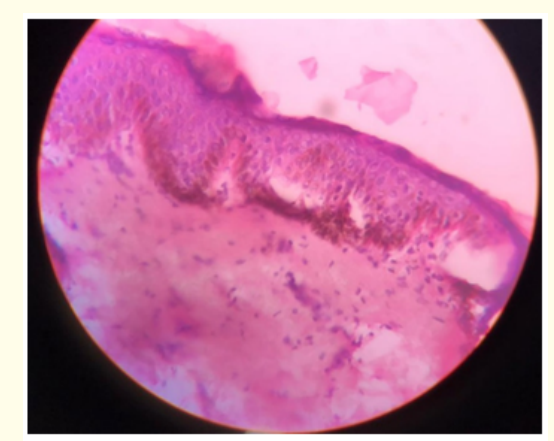

Figure 3: Microscopy showing collagen deposition and lymphocyte infiltration.

Child was diagnosed with superficial circumscribed Morpea based on symptoms, clinical findings and biopsy report. This type of Morphea is rare at the age of 2 and is usually seen in children of 5 - 7 years old. Initially child was started with fluticasone propionate $0.05 \%$ cream and tacrolimus $0.03 \%$ ointment for 3 weeks. As there was no improvement, calcitriol 3 mcg ointment (Psorafuse) with tacrolimus $0.03 \%$ ointment was prescribed for the next 2 weeks.

Biopsy was done following this and the diagnosis of Morphea was confirmed. Syrup Prednisolone was prescribed for 1 week, tapered over one week and calcitriol 3 mcg ointment (psorafuse) and pimecrolimus $1 \%$ was continued for next two weeks and only pimecrolimus thereafter. No antifibrotic drugs were used in the course of the treatment Though there was no immediate changes in Alopecia, the hypo pigmented lesions started getting healed without any further complications.

\section{Discussion}

In Morphea, the lesions are generally limited to the skin and subcutaneous fatty tissue, but they can reach up to muscular fascia, muscle tissue, joint synovia, tendons, and bone marrow too. Other symptoms which can accompany skin involvement are arthralgia, synovitis, and infrequent ipsilateral uveitis, or even Raynaud's phenomenon. Visceral involvements, which generally affect pulmonary function or esophageal motility, is considered as an attribute of systemic sclerosis and is mostly rare in patients with Morphea. Therefore, prognosis of patients with Morphea is usually good [4,5].

According to the Mayo Clinic classification, there are additional subtypes of Morphea: Linear Morphea, Plaque Morphea, Bullous Morphea, Deep Morphea (including disabling pansclerotic Morphea of children), and generalized Morphea (comprising eosinophilic fasciitis) [3]. The Generalized Morphea is the most severe form of localized Morphea. The following clinical diagnostic criteria for this subtype of Morphea must be fulfilled: four or more lesions larger than $3 \mathrm{~cm}$ in diameter or involvement of two or more of the seven body areas (the head and neck, the right and left upper extremities, the anterior and posterior trunks, and the right and left lower extremities) [6,7].

Imaging of Morphea lesions may not show any specific findings, but involvement of the skin, subcutaneous fatty tissue, and muscle fasciae can be easily recognized on MRI. Radio imaging is required for accurate classification of Morphea and the treatment. Histopathological examination of the lesion reveals different degrees of collagenization of the dermis and extension of fibrous tissue into the subcutaneous fat, fascia, musculature, and infrequently, into the bone marrow, at the later course of the disease. Yet, depth of infiltration by collagen bundles is expected primarily in the deep and generalized types of Morphea. Autoimmune abnormalities of localized scleroderma have been well documented during the past few decades, and recently this disease has been considered to have an autoimmune background because of the high frequency of antinuclear antibodies encountered [8].

Conditions that resemble Morphea include mainly connective tissue diseases with skin, fascial, and musculature involvement such as systemic sclerosis. It's generally excluded first in all patients presenting with localized Morphea, dermatomyositis, systemic lupus erythematosus, overlapping syndromes, and other forms of fasciitis [9]. Rarer differential diagnoses are pseudoscleroderma related to inflammatory syndromes induced by toxins, radiation, silicon, drugs, or paraffin implants and graft-versus- host disease (GVHD) of the skin. 
There are a wide range of therapeutic options available for the treatment of Morphea but large scale controlled trials on this regard are rare. The choice of treatment modality is subjected to the depth and extent of involvement and the clinical activity of the disease.

Immunosuppressive therapies such as oral corticosteroids or methotrexate can be useful in the inflammatory stage of the disease. Topical steroids are the most commonly favored first line treatment for cases of Morphea with limited involvement. Active disease without deep inflammation is initially treated by a 3-month course of moderate- to high-potent topical corticosteroids. Few studies have found out Intralesional steroid injections are helpful for cessation of progression in localized active disease. Topical tacrolimus $0.1 \%$ ointment with or without occlusion can be considered for treatment of active plaque Morphea. There are studies showing Topical calcipotriol $0.005 \%$ effective in improving Morphea lesions previously unresponsive to topical steroids but weekly calcipotriol dose should not exceed $50 \mathrm{~g} / \mathrm{m}^{2}$ [10,11]. Topical imiquimod $5 \%$ has also been studied as an alternative therapeutic option for the treatment of plaque Morphea. Lesions with deeper involvement or causing functional impairments are treated with systemic therapies. Methotrexate has the most evidence for efficacy, proven by many randomized controlled studies [12].

Phototherapy is the first-line treatment of choice for extensive or rapidly progressing disease without deep involvement and can also be considered for patients with partial involvement that are unresponsive to topical treatment [13]. In patients showing no deeper infiltration, significant progress can be achieved by both psoralen ultraviolet A-range photochemotherapy and ultraviolet A-1 (narrowband ultraviolet radiation) therapy. Nevertheless, fascial, muscular, and osseous involvement in generally requires systemic therapy.

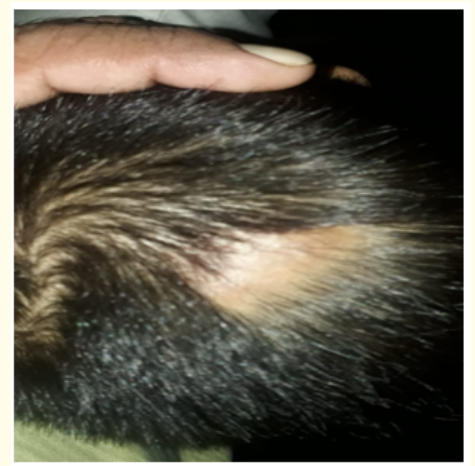

Figure 4: Scalp - post treatment.

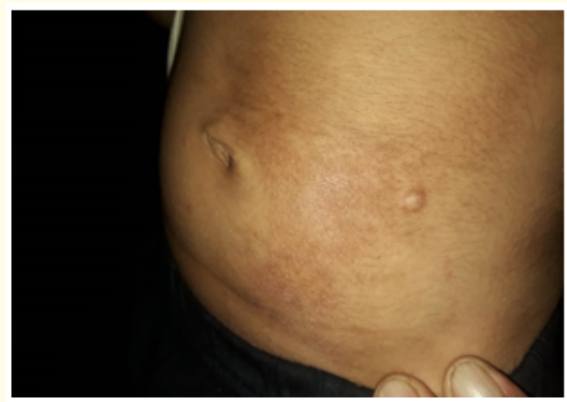

Figure 5: Hypo pigmented patch around umbilicus- Post treatment.

\section{Follow-up recommendations}

The child was advised for a follow-up interval of two weeks, during the initial course of the treatment. Adequate response for the treatment was seen after a period of 4 - 5 months of treatment. Now the patient needs be evaluated by dermatologist for skin elasticity and any skin color changes and a periodic evaluation from rheumatologist to rule out any connective tissue disorder during follow up. No investigations are generally required during follow up period unless there are aggravation of symptoms. In the long run, to rule out systemic sclerosis, chest x-ray may be needed. Treatment may be needed lifelong since it's an auto immune disorder. The patient was recommended for follow-up every 6 months and needs to continue tacrolimus $0.03 \%$ ointment for 2 years.

\section{Conclusion}

Although Morphea causes functional and aesthetic impairment, it is rarely life-threatening. Morphea may be self-limited, but frequently has a remitting relapsing or chronic course producing significant disease burden over time. A correlation of clinical and histopathological findings is important to reach a correct diagnosis, ensure appropriate treatment, and monitor for comorbid conditions. Early diagnosis and treatment is crucial to minimize potential sequela.

\section{Conflict of Interest}

None.

\section{Bibliography}

1. Fett NM. "Morphea (localized scleroderma)". JAMA Dermatology 149 (2013): 1124.

2. Kauer F., et al. "Nodular Morphea". Dermatology 218 (2009): 63-66.

3. Peterson LS., et al. "Classification of Morphea (localized scleroderma)”. Mayo Clinic Proceedings 70 (1995): 1068-1076. 
4. Takehara $\mathrm{K}$ and Sato S. "Localized scleroderma is an autoimmune disorder". Rheumatology 44.3 (2005): 274-279.

5. Dehen L., et al. "Internal involvement in localized scleroderma”. Medicine (Baltimore) 73 (1994): 241-245.

6. Devidayal Singh S., et al. "Disabling pansclerotic Morphea of childhood and hypogammaglobulinemia: a curious association". Rheumatology International 21 (2002): 158-160

7. Doede T., et al. "Pansclerotic Morphea in childhood: a case report”. Pediatric Surgery International 19 (2003): 406-408.

8. Sato S., et al. "Clinical characteristics associated with antihistone antibodies in patients with localized scleroderma". Journal of the American Academy of Dermatology 31 (1994): 567571.

9. Al Attia HM., et al. "A localized Morphea/idiopathic polymyositis overlap". Clinical Rheumatology 15 (1996): 307-309.

10. Cunningham BB., et al. "Topical calcipotriene for Morphea/ linear scleroderma". Journal of the American Academy of Dermatology 39 (1998): 211-215.

11. Tay YK. "Topical calcipotriol ointment in the treatment of Morphea". Journal of Dermatological Treatment 14 (2003): 219221.

12. Li SC., et al. "Initial Results from a Pilot Comparative Effectiveness Study of Three Methotrexate-Based Consensus Treatment Plans for Juvenile Localized Scleroderma". Journal of Rheumatology (2019): jrheum.190311.

13. Florez-Pollack S., et al. "Morphea: Current concepts". Clinical Dermatology 36 (2018): 475-486.

\section{Assets from publication with us}

- Prompt Acknowledgement after receiving the article

- Thorough Double blinded peer review

- Rapid Publication

- Issue of Publication Certificate

- High visibility of your Published work

Website: www.actascientific.com/

Submit Article: www.actascientific.com/submission.php

Email us: editor@actascientific.com

Contact us: +919182824667

Citation: Edwin Dias., et al. "A Case Report of Morphea in a 2 Years Old Child". Acta Scientific Paediatrics 4.4 (2021): 66-70. 